and MMP13 in rat chondrocytes after IL1 $\beta$ stimulation. In vitro anti-inflammatory effect was determined by measuring the secretion of IL6, IL8, IL1 $\beta$, TNFa and IFN $\gamma$ by human monocytes. In vivo, the pharmacodynamic of our small molecules was assessed after intravenous and oral administration. Therapeutic efficacy of a compound was then evaluated in a collagen-induced arthritis model in DBA $1 / \mathrm{J}$ mice. In this model, measures of the arthritis index score, body weight, plasma level of TNFa, IL6, IL8 and IL1 $\beta$ and histological evaluation of cartilage erosion were performed.

Results: Our new series of small molecules are potent PAR2 inhibitors (IC50<1nM in calcium assay) with some selectivity over PAR1 and PAR4. Our compounds significantly inhibited PAR2 mediated recruitment of $\mathrm{Gz}, \mathrm{Gq}, \mathrm{G} 13$, G14 and G15. However, surprisingly, these small molecules had no effect on B arrestin 2, EPAC, Gi2 and GoB demonstrating that they are biased inhibitors. The effect of our compounds on PAR2 signalling was clearly different from 3 already existing PAR2 inhibitors described in the literature (I-117, AZ3451 and P2pal-18s). We compared the in vitro anti-hypertrophic effect on chondrocyte and anti-inflammatory effect on monocytes of these compounds to determine the importance of PAR2 signalling pathways in these cellular functions. In vivo, our small molecules had good bioavailability after oral administration of $10 \mathrm{mg} / \mathrm{kg}$ in mice (clearance $=$ $0.038 \mathrm{~L} / \mathrm{h} / \mathrm{kg} ; \mathrm{T} 1 \frac{1}{2}=9.9 \mathrm{~h} ; \mathrm{AUC}=162564 \mathrm{ng} \cdot \mathrm{h} / \mathrm{mL} ; \mathrm{Cmax}=9005 \mathrm{ng} / \mathrm{mL}$ ). The in vivo therapeutic efficacy of a biased PAR2 inhibitor in a model of collagen-induced arthritis will be presented.

Conclusion: Our results show the potency of biased PAR2 inhibitors to reduce both the inflammation and cartilage erosion in rheumatoid arthritis. They confirm the huge potential of PAR2 as a therapeutic target and unravel the relevance of biased antagonism of this receptor to treat rheumatic diseases.

References:

[1] McCulloch et al., Frontiers in Endocrinology, 2018; ${ }^{2}$ Hollenberg et al., British

Journal of Pharmacology, 2014; ${ }^{3}$ Sharma et al., Genes and Immunity, 2015;

${ }^{4}$ Rayees et al., Cell Reports, 2019

Disclosure of Interests: Thibaut Brugat Employee of: Domain Therapeutics, Baptiste Rugeri Employee of: Domain Therapeutics, Gaël Hommet Employee of: Domain Therapeutics, Alexia Dumont Employee of: Domain Therapeutics, Luc Baron Employee of: Domain Therapeutics, Célia Halter Employee of: Domain Therapeutics, Meriem Sémache Employee of: Domain Therapeutics, Arturo Mancini Employee of: Domain Therapeutics, Camille Amalric Employee of: Domain Therapeutics, Marie Giambelluco Employee of: Domain Therapeutics, Nathalie Lenne Employee of: Domain Therapeutics, Marjorie Sidhoum Employee of: Domain Therapeutics, Christel Franchet Employee of: Domain Therapeutics, Stanislas Mayer Employee of: Domain Therapeutics, Xavier Leroy Employee of: Domain Therapeutics, Stephan Schann Employee of: Domain Therapeutics DOI: 10.1136/annrheumdis-2020-eular.5353

\section{OP0237 THROMBOEMBOLIC SAFETY PROFILE OF TOFACITINIB AND BARICITINIB: AN ANALYSIS OF WHO VIGIBASE}

E. Vallejo-Yagüe ${ }^{1}$, S. Weiler ${ }^{1,2}$, A. M. Burden ${ }^{1} .{ }^{1}$ ETH Zürich, Department of Chemistry and Applied Biosciences, Zürich, Switzerland; ${ }^{2}$ National Poisons Information Centre, Tox Info Suisse, Associated Institute of the University of Zurich, Zurich, Switzerland

Background: The Janus Kinase (JAK) inhibitors tofacitinib and baricitinib are new targeted treatments for rheumatoid arthritis. Recent concerns regarding the risk of thrombosis have led to warnings by the European Medicines Agency (EMA) and the Food and Drug Administration (FDA) ${ }^{1,2}$.

Objectives: To examine the safety reporting of tofacitinib and baricitinib, with focus on thromboembolic events.

Methods: Individual case safety reports (ICSRs) for tofacitinib and baricitinib were retrieved from the World Health Organization (WHO) global database (VigiBase) in April 2019. The primary outcomes were deep vein thrombosis (DVT) and pulmonary thrombosis (PT) or pulmonary embolism (PE). A disproportionality analysis was conducted by estimating the reporting odds ratio (ROR) and $95 \%$ confidence intervals (Cls) to compare the observed versus expected reporting ratio of DVT or PTIPE for tofacitinib or baricitinib. The ROR were calculated worldwide and stratifying by reporting from Europe or the US. In a secondary analysis, further thrombotic-related outcomes were investigated.

Results: In both tofacitinib $(n=40,017)$ and baricitinib $(n=2,138)$ ICSRs, patients with reported DVT or PTIPE were older and had higher reporting of pro-thrombotic medications (e.g., contraceptives) or indicators of thromboembolic risk (i.e., antithrombotic treatment). The use of tofacitinib was associated with a significant increased reporting for DVT (ROR: 2.37 95\% Cl 1.23-4.56) and PTIPE (ROR $2.3895 \% \mathrm{Cl} 1.45-3.89$ ) in Europe. In the US, tofacitinib was only associated with an elevated reporting of PT (ROR: $2.05 \% \mathrm{Cl}$ 1.45-2.90). Baricitinib was associated with a 3-fold increased risk of reporting for DVT (ROR: $3.4795 \% \mathrm{Cl}$ 2.18-5.52) or PTIPE (ROR: $3.4495 \% \mathrm{Cl}$ 2.43-4.88) in Europe, which accounted for $97 \%$ of all baricitinib ICSRs. Secondary thrombotic-related outcomes were poorly reported overall in VigiBase.

Conclusion: This study supports the cautious use of JAK inhibitors in patients with rheumatoid arthritis who have a high thrombotic risk profile. Moreover, a potential class effect of JAK inhibitors cannot be ruled out.

\section{References:}

[1] FDA Drug Safety Communication. Safety trial finds risk of blood clots in the lungs and death with higher dose of tofacitinib (Xeljanz, Xeljanz XR) in rheumatoid arthritis patients; FDA to investigate. Food and Drug Administration (FDA) http://www.fda.gov/drugs/drug-safety-and-availability/ safety-trial-finds-risk-blood-clots-lungs-and-death-higher-dose-tofacitinibxeljanz-xeljanz-xr (2019).

[2] EMA confirms Xeljanz to be used with caution in patients at high risk of blood clots. EMA/608520/2019. https://www.ema.europa.eu/en/documents/ referral/xeljanz-article-20-procedure-ema-confirms-xeljanz-be-used-caution-patients-high-risk-blood-clots_en.pdf (2019).

Acknowledgments: We are thankful to every pharmacovigilance centre and contributor to the WHO Programme for International Drug Monitoring and VigiBase.

While the authors used data from the VigiBase, the WHO global database of ICSRs as a source of information, the conclusions do not represent the opinion of the Uppsala Monitoring Centre (UMC) or the WHO.

Disclosure of Interests: Enriqueta Vallejo-Yagüe Employee of: Synovo GmbH 2012-2018 (not related to this abstract), Stefan Weiler Consultant of: Gedeon-Richter for drug safety 2017 (not related to this abstract), Andrea Michelle Burden: None declared DOI: 10.1136/annrheumdis-2020-eular.2823

\begin{tabular}{|l|l}
\hline OP0238 & RISK OF HERPES ZOSTER IN PATIENTS WITH \\
& RHEUMATOID ARTHRITIS UNDER BIOLOGICAL, \\
& TARGETED SYNTHETIC, AND CONVENTIONAL \\
& SYNTHETIC DMARD TREATMENT
\end{tabular}

A. Strangfeld $^{1}$, I. Redeker ${ }^{1}$, J. Kekow ${ }^{2}$, G. R. Burmester ${ }^{3}$, J. Braun ${ }^{4}$, A. Zink ${ }^{1,3}$. ${ }^{1}$ German Rheumatism Research Centre, Berlin, Germany; ${ }^{2}$ Scientific Advisory Board, Vogelsang-Gommern, Germany; ${ }^{3}$ Charité University Medicine, Berlin, Germany; ${ }^{4}$ Rheumatologist, Herne, Germany

Background: The risk of herpes zoster $(\mathrm{HZ})$ is higher in patients with rheumatoid arthritis (RA) than in the general population. This risk is further increased with biologic disease-modifying anti-rheumatic drugs (bDMARDs) such as tumour necrosis factor inhibitors (TNFi) and targeted synthetic (ts)DMARDs such as Janus kinase inhibitors (JAKi) compared to patients taking conventional synthetic (cs)DMARDs such as methotrexate (MTX).

Objectives: To compare incidence rates of $\mathrm{HZ}$ in RA patients under treatment with bDMARDs, tsDMARDs and csDMARDs with different modes of action and to find potential risk factors.

Methods: Data of patients enrolled in the German biologics register RABBIT from 2007 onwards with the start of a bDMARD, tsDMARD or a change in csDMARD treatment were analysed. Patients were included when at least one follow-up documentation was available. All HZ events reported until 30 April 2019 were identified and assigned to treatments administered within the 3 month period prior to the $\mathrm{HZ}$ event. Crude incidence rates (IR) of $\mathrm{HZ}$ were calculated per 1,000 patient years (py). Cox regression was applied to investigate risk factors for the occurrence of $\mathrm{HZ}$ with and without inverse probability weights (IPW) to adjus for confounding by indication.

Results: Data of 12,470 patients $(53,218$ py of observation) were included in the analysis. A total of $452 \mathrm{HZ}$ cases in 433 patients were reported, of which 52 events were serious. The crude IRs per 1,000 py are illustrated by Figure. Adjusted for age, sex, and glucocorticoid use, a significantly increased risk was observed for treatment with monoclonal TNF antibodies (hazard ratio [HR], 1.55 [95\% Cl, 1.21-2.00]), B-cell targeted therapies ( $\mathrm{HR}, 1.45$ [95\% Cl, 1.07-1.97]), and tsDMARDs (HR, 3.55 [95\% Cl, 2.33-5.41]). Treatment with soluble TNF receptors, T-cell co-stimulation modulator, and IL-6 inhibitors were not significantly associated (Table). Adjustment with IPW amplified the effect and treatment with T-cell co-stimulation modulator and IL-6 inhibitors were also significantly associated with a higher risk compared to csDMARD treatment (Table).

Conclusion: This is the first analysis in a European prospective cohort study comparing the incidence rates and risk of $\mathrm{HZ}$ in $\mathrm{RA}$ patients under treatment with six different modes of action within one cohort to cSDMARD treatment. We found a significant association between $\mathrm{HZ}$ and treatment with JAKi. Our results also confirm a higher risk for monoclonal TNF antibodies and show a similar result for the T-cell co-stimulation modulator and B-cell targeted therapies. This study clearly supports systematic $\mathrm{HZ}$ vaccination of RA patients. 